\title{
CO bands in V4334 Sgr (Sakurai's Object): The ${ }^{12} \mathrm{C} /{ }^{13} \mathrm{C}$ ratio
}

\author{
Ya. V. Pavlenko ${ }^{1}$, T. R. Geballe ${ }^{2}$, A. Evans ${ }^{3}$, B. Smalley ${ }^{3}$, S. P. S. Eyres ${ }^{4}$, V. H. Tyne ${ }^{3}$, and L. A. Yakovina ${ }^{1}$ \\ ${ }^{1}$ Main Astronomical Observatory, Academy of Sciences of the Ukraine, Golosiiv Woods, Kyiv-127, 03680 Ukraine \\ 2 Gemini Observatory, 670 N. A'ohoku Place, Hilo, Hi 96720, USA \\ 3 Astrophysics Group, School of Chemistry \& Physics, Keele University, Keele Staffordshire, ST5 5BG, UK \\ ${ }^{4}$ Centre for Astrophysics, University of Central Lancashire, Preston, Lancashire PR1 2HE, UK
}

Received 19 January 2004 / Accepted 26 February 2004

\begin{abstract}
We present the results of our analysis of a high resolution $(R \simeq 30000)$ infrared spectrum of V4334 Sgr (Sakurai's Object) around the first overtone $\mathrm{CO}$ bands, obtained in $1998 \mathrm{July}$. The ${ }^{12} \mathrm{CO}$ and ${ }^{13} \mathrm{CO}$ bands are well-resolved, and we compute synthetic hydrogen-deficient model atmosphere spectra to determine the ${ }^{12} \mathrm{C} /{ }^{13} \mathrm{C}$ ratio. We find ${ }^{12} \mathrm{C} /{ }^{13} \mathrm{C} \simeq 4 \pm 1$, consistent with the interpretation of V4334 Sgr as an object that has undergone a very late thermal pulse.
\end{abstract}

Key words. stars: individual: V4334 Sqr - stars: individual: Sakurai’s Object - stars: atmospheric parameters stars: abundances

\section{Introduction}

V4334 Sgr (Sakurai's Object) is a low mass $\left(\sim 0.6-0.8 M_{\odot}\right.$; Herwig 2001) star undergoing a very late thermal pulse (VLTP). After traversing the "knee" on the post-asymptotic giant branch (AGB) evolutionary track, the star experiences a final He flash as it evolves down the white dwarf cooling track: it becomes a "born-again" AGB star (see Herwig 2001; Lawlor \& MacDonald 2003)

A key factor in confirming this scenario is the determination of elemental abundances and isotopic ratios in the surface material. Asplund et al. (1999) have used optical spectroscopy to determine the abundance of a range of species; they found V4334 Sgr to be hydrogen-deficient, with other elemental abundances close to those of the R $\mathrm{CrB}$ stars; $\mathrm{R} \mathrm{CrB}$ stars. They also used the $\mathrm{C}_{2}(1-0)$ and (0-1) Swan bands at 4740 and $5635 \AA$ respectively to determine that the ${ }^{12} \mathrm{C} /{ }^{13} \mathrm{C}$ ratio was somewhere in the range $1.5-5$, although they did not give an actual value.

Pavlenko \& Geballe (2002) modelled the spectral energy distribution of V4334 Sgr in the near-IR $(\lambda \lambda 1-2.5 \mu \mathrm{m})$. They broadly confirmed the results of Asplund et al. (1999), but found some evidences of higher $\mathrm{C}$ abundance, by a factor $\sim 2$. Pavlenko \& Geballe also noted the presence of hot dust longward of $\sim 2 \mu \mathrm{m}$.

The ${ }^{12} \mathrm{C} /{ }^{13} \mathrm{C}$ ratio is of fundamental importance in understanding the VLTP process, as it is a reflection of the relative importance of ${ }^{12} \mathrm{C},{ }^{13} \mathrm{C}$ processing and dredge-up. In this Letter we use echelle observations of the first overtone vibrationrotation features of CO, obtained in 1998 July while the star was still visible, to determine the ${ }^{12} \mathrm{C} /{ }^{13} \mathrm{C}$ ratio in V4334 Sgr.

Send offprint requests to: Ya. V. Pavlenko, e-mail: yp@mao.kiev. ua

\section{Observation}

The data were obtained with the facility spectrometer CGS4 (Mountain et al. 1990) at the $3.8 \mathrm{~m}$ UK Infrared Telescope on UT 1998 July 7. CGS4 was configured with a $256 \times$ $256 \mathrm{InSb}$ array, its $311 \mathrm{~mm}^{-1}$ echelle and a $0.45^{\prime \prime}$ slit, which provided a resolving power of $\sim 30000$. The spectrum was sampled every 0.4 resolution element. Five echelle settings were used to continuously cover the wavelength range $2.32-2.38 \mu \mathrm{m}$, an interval containing numerous ${ }^{12} \mathrm{CO}$ and ${ }^{13} \mathrm{CO}$ lines, including three bandheads of ${ }^{12} \mathrm{CO}(\delta v=3 \rightarrow$ $1,4 \rightarrow 2,5 \rightarrow 3)$ and two of ${ }^{13} \mathrm{CO}(\delta v=2 \rightarrow 0,3 \rightarrow 1)$. Each of the five spectral segments was observed for a total of $8 \mathrm{~min}$, with individual data points being observed for $4 \mathrm{~min}$. A spectrum of the A2V star HR 6378 was obtained just prior to each spectrum of V4334 Sgr and at similar airmass. The raw spectra of V4334 Sgr and HR 6378 were extracted, reduced, and ratioed using standard techniques. Wavelength calibration was achieved using some of the multitude of telluric absorption lines in the spectra of the calibration star, and is accurate to $\pm 3 \mathrm{~km} \mathrm{~s}^{-1}$; the wavelength scale is in vacuum. The ratioed spectral segments slightly overlap one another and were scaled and adjoined to provide the final spectrum used in the analysis.

\section{Procedure}

\subsection{Spectral synthesis}

We have used the technique of synthetic spectra to carry out our analysis of the IR spectrum of V4334 Sgr. We computed the spectra in the classical framework, assuming LTE, planeparallel media, and no sinks and sources of energy in the atmosphere. The transfer of energy is provided by the radiation 


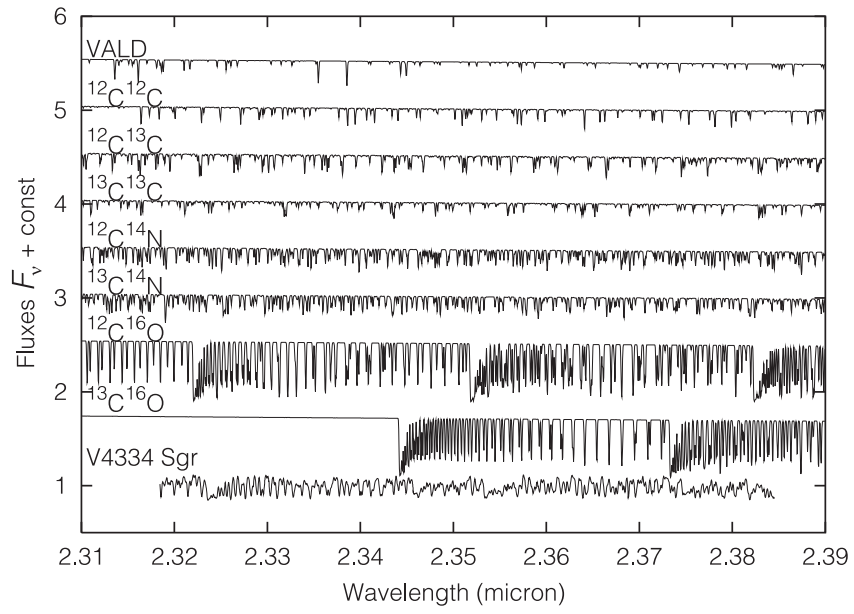

Fig. 1. Theoretical spectra of different species in the spectra region convolved by Gaussian of half-width $5 \times 10^{-6} \mu \mathrm{m}$. Synthetic spectra were computed for ${ }^{12} \mathrm{C} /{ }^{13} \mathrm{C}=1$.

field and by convection. Strictly speaking, none of these assumptions is completely valid in the atmosphere of V4334 Sgr because, in all probability, we see only the pseudophotosphere, i.e. the outermost part of the expanding envelope. However, we suggest that none of these assumptions is of crucial importance for the spectrum formation processes.

A grid of LTE synthetic spectra was computed for ${ }^{12} \mathrm{C} /{ }^{13} \mathrm{C}$ ratios of $1,2,3,4,5,6,7,8,9$, 10, model atmosphere parameters $T_{\text {eff }}, \log g=5250,0.0$ (Pavlenko \& Dürbeck 2002), in the wavelength range $2.31-2.39 \mu \mathrm{m}$ and with wavelength step $0.00002 \mu \mathrm{m}$, using the WITA612 code (Pavlenko 1997). We used abundances as given by Asplund et al. (1999), scaled such that $\sum N_{i}=1$, and with $\mathrm{N}, \mathrm{O}$ abundances of -2.52 , -2.02 respectively, and two abundances of carbon, namely $\log N(\mathrm{C})=-1.05$ and $\log N(\mathrm{C})=-1.62$; the first case corresponds to a carbon-rich model atmosphere (see Asplund et al. 1999 for a discussion of the carbon problem). As the determination of the ${ }^{12} \mathrm{C} /{ }^{13} \mathrm{C}$ ratio in stellar atmospheres depends on $V_{\mathrm{t}}$ (see Pavlenko et al. 2003), we determined ${ }^{12} \mathrm{C} /{ }^{13} \mathrm{C}$ for two values of the microturbulent velocity, $V_{\mathrm{t}}=3$ and $6 \mathrm{~km} \mathrm{~s}^{-1}$.

A set of continuum opacity sources from ATLAS9 (Kurucz 1993) was used. Additionally, all the major sources of opacity in our spectral region of interest were taken into account:

(i) bound-free absorption by $\mathrm{C}$ I, N I, O I atoms computed by Pavlenko \& Zhukovska (2003) for the TOPBASE crosssections (Seaton et al. 1992);

(ii) ${ }^{12} \mathrm{C}^{16} \mathrm{O},{ }^{13} \mathrm{C}^{16} \mathrm{O}$ lines from Goorvitch (1994);

(iii) ${ }^{12} \mathrm{C}^{12} \mathrm{C},{ }^{12} \mathrm{C}^{13} \mathrm{C},{ }^{13} \mathrm{C}^{13} \mathrm{C},{ }^{12} \mathrm{C}^{14} \mathrm{~N}$ and ${ }^{13} \mathrm{C}^{14} \mathrm{~N}$ lines from Kurucz (1993);

(iv) atomic lines from VALD (Kupka et al. 1999).

We adopt the Voigt profile $H(a, v)$ for the shape of each line; damping constants $a=\left(\gamma_{2}+\gamma_{4}+\gamma_{6}\right) /\left(4 \times \pi \times \Delta \nu_{D}\right)$ were computed using data from various databases (Kurucz 1993; Kupka et al. 1999), or computed following an approximation of Unsöld (1955). The contributions of the various molecular and atomic species to the total opacity in the $2.31-2.39 \mu \mathrm{m}$ spectral region are shown in Fig. 1, from which we see that absorption by $\mathrm{CO}$ dominates. Finally, bearing in mind the high luminosity of V4334 Sgr $\left(\sim 10^{4} L_{\odot}\right.$; Herwig 2001), we adopt $\log g=0$.

\subsection{The effect of circumstellar dust}

Pavlenko \& Geballe (2002) have shown that the $2 \mu \mathrm{m}$ spectral region of V4334 Sgr is affected by emission from the hot dust known to envelop V4334 Sgr at this time, and which most likely formed at some 20 stellar radii from the stellar atmosphere (Kipper 1999; Tyne et al. 2002). The dust shell first appeared in 1997 March and, by 1998 August, its presence was well established photometrically (Kerber et al. 1999; Kipper 1999) and spectroscopically (Tyne et al. 2000, 2002).

The dust emission veils the $\mathrm{CO}$ absorption lines, and to estimate its effect we suppose that the dust emission adds some continuum flux in our spectral region, effectively reducing the residual fluxes $r_{v}=F_{v}^{1+\mathrm{c}} / F_{v}^{\mathrm{c}}$ (and hence equivalent widths) of the absorption lines; here $F_{v}^{1+\mathrm{c}}$ and $F_{v}^{\mathrm{c}}$ are fluxes in the lines and continuum respectively. We describe this by the factor

$r_{v}=\left(r_{v}^{0}+\epsilon_{0}\right) /\left(1+\epsilon_{0}\right)$,

where $r_{v}^{0}$ and $\epsilon_{0}$ are respectively the residual flux computed for the "dust-free" case, and the relative contribution of the dust emission; the parameter $\epsilon_{0}$ is determined from comparison of computed and observed spectra.

\subsection{Fitting procedure}

To determine the best fit parameters, we compare the observed residual fluxes $r_{v}$ with computed values following the scheme of Jones et al. (2002) and Pavlenko \& Jones (2002). We let

$r_{v}^{x}=\int r_{v}^{y} \times G(x-y) * \mathrm{~d} y$

where $r_{v}^{y}$ and $G(x-y)$ are respectively the residual fluxes computed by WITA612 and the broadening profile; we adopt a Gaussian for the latter. We then find the minima of the 3D function

$S\left(f_{\mathrm{s}}, f_{\mathrm{h}}, f_{\mathrm{g}}\right)=\sum\left(1-f_{\mathrm{h}} \times r^{\mathrm{synt}} / r^{\mathrm{obs}}\right)^{2}$,

where $f_{\mathrm{s}}, f_{\mathrm{g}}, f_{\mathrm{g}}$ are respectively the wavelength shift, normalisation factor, and half-width of $G(x-y)$. The parameters $f_{\mathrm{s}}, f_{\mathrm{h}}$ and $f_{\mathrm{g}}$ are determined by the minimization procedure for every computed spectrum. Then, from the grid of the better solutions for a given ${ }^{12} \mathrm{C} /{ }^{13} \mathrm{C}$ and $\epsilon_{0}$, we choose the best-fitting solution.

\section{Results}

On July 7 the Earth's orbital motion redshifted the spectrum of V4334 Sgr by $8 \mathrm{~km} \mathrm{~s}^{-1}$. Thus the shift of $+112 \mathrm{~km} \mathrm{~s}^{-1}$ that we needed to apply means that the heliocentric velocity of the photosperic $\mathrm{CO}$ was $+104 \pm 3.2 \mathrm{~km} \mathrm{~s}^{-1}$, where the uncertainty includes $\pm 3 \mathrm{~km} \mathrm{~s}^{-1}$ for the wavelength scale, and $\pm 1 \mathrm{~km} \mathrm{~s}^{-1}$ in our shift. While this is close to the known heliocentric velocity of V4334 Sgr, $+115 \mathrm{~km} \mathrm{~s}^{-1}$ (Dürbeck \& Benetti 1996), it may also imply that the photospheric layers in which the $\mathrm{CO}$ was located were expanding at $\sim 10 \mathrm{~km} \mathrm{~s}^{-1}$. 

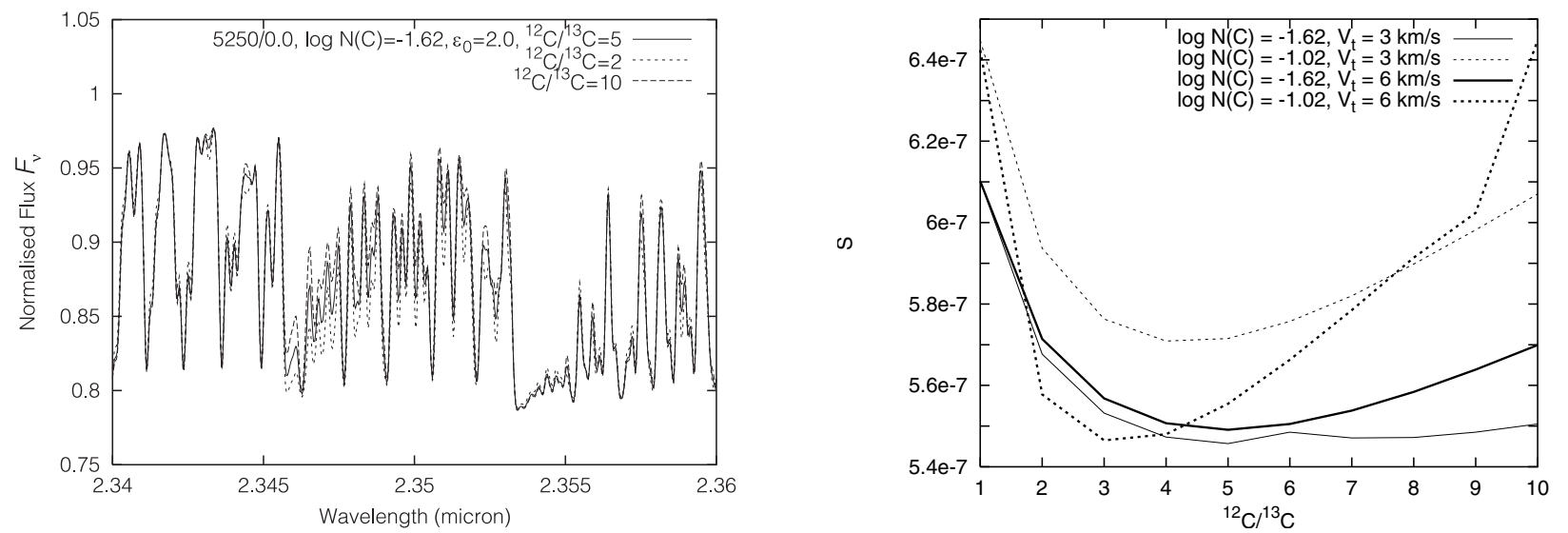

Fig. 2. a) Dependence of computed spectra on ${ }^{12} \mathrm{C} /{ }^{13} \mathrm{C}$ for values of $T_{\text {eff }}, \log g$ and $\epsilon_{0}$ indicated. b) Dependence of the parameter $S$ on ${ }^{12} \mathrm{C} /{ }^{13} \mathrm{C}$ for values of $\log N(\mathrm{C})$ and $V_{\mathrm{t}}$ indicated.

Table 1. Atmospheric and dust parameters for V4334 Sgr in 1998 July.

\begin{tabular}{lcccc}
\hline \hline $\log N(\mathrm{C})$ & \multicolumn{2}{c}{-1.05} & \multicolumn{2}{c}{-1.62} \\
$V_{\mathrm{t}}\left(\mathrm{km} \mathrm{s}^{-1}\right)$ & 3 & 6 & 3 & 6 \\
\hline Shift $\left(10^{-4} \mu \mathrm{m}\right)$ between & & & & \\
observed and computed spectra & 8.6 & 8.6 & 8.6 & 8.6 \\
Normalisaton factor $f_{\mathrm{h}}$ & 0.96 & 0.96 & 0.96 & 0.96 \\
$F W H M\left(10^{-4} \mu \mathrm{m}\right)$ & 1.56 & 1.4 & 1.8 & 1.4 \\
$\epsilon_{0}$ & 1.4 & 2.0 & 1.8 & 2.4 \\
${ }^{12} \mathrm{C} /{ }^{13} \mathrm{C}$ & 4 & 3 & $(5,8)$ & 5 \\
\hline
\end{tabular}

Our results show that ${ }^{12} \mathrm{C} /{ }^{13} \mathrm{C}$ depends on several parameters. Figure $2 \mathrm{a}$ shows the dependence of the synthetic spectra on the ${ }^{12} \mathrm{C} /{ }^{13} \mathrm{C}$ ratio for $\epsilon_{0}=2.0$, while Fig. $2 \mathrm{~b}$ shows the dependence of the best-fit parameter $S$ on the ${ }^{12} \mathrm{C} /{ }^{13} \mathrm{C}$ ratio for a variety of $\log N(\mathrm{C})$ and $V_{\mathrm{t}}$ values. Both the ${ }^{12} \mathrm{C}^{16} \mathrm{O}$ and ${ }^{13} \mathrm{C}^{16} \mathrm{O}$ bands are strong in the spectra due to the high carbon abundance; the computed central intensities of the strongest lines are $\sim 0.4$ of the normalized continuum.

Using our fitting procedure we found a clear dependence of the spectra on ${ }^{12} \mathrm{C} /{ }^{13} \mathrm{C}, V_{\mathrm{t}}$ and $\epsilon_{0}$. The best-fit synthetic spectra for our two assumed $\mathrm{C}$ abundances are shown in Figs. 3a,b; the corresponding parameters are given in Table 1. Note that there are two possible solutions for $\left[\log N(\mathrm{C}), V_{\mathrm{t}}\right]=[-1.62,3]$ (see Fig. 2b). Figures $4 a, b$ shows the $2 \mathrm{D}$-dependence of the fit parameter $S$ on ${ }^{12} \mathrm{C} /{ }^{13} \mathrm{C}$ and $\epsilon_{0}$. We effectively found two families of solutions, differing by their ${ }^{12} \mathrm{C} /{ }^{13} \mathrm{C}$ ratios and $\epsilon_{0}$ values. An alternative interpretation is that there is an uncertainty in our results dependent on the uncertainties in the input parameters.

In general, a mictoturbulent velocity $V_{\mathrm{t}}=6 \mathrm{~km} \mathrm{~s}^{-1}$ is most likely for a supergiant atmosphere and our results for $V_{\mathrm{t}}=$ $3 \mathrm{~km} \mathrm{~s}^{-1}$, presented in Table 1, are included primarily to illustrate the $V_{\mathrm{t}}$-dependence of our results. We see from Figs. $2 \mathrm{~b}$ and 4 , and Table 1 , that a ${ }^{12} \mathrm{C} /{ }^{13} \mathrm{C}$ ratio of $4 \pm 1$ is consistent with the data.

\section{Discussion}

One of the major challenges posed by V4334 Sgr has been the extreme rapidity with which it has evolved following the VLTP. Herwig (2001) has addressed this problem by supposing that the element mixing efficiency in the He flash convection zone during the VLTP is much smaller than predicted by mixing length theory. Herwig defines the parameter $f=D_{\mathrm{MLT}} / D_{\mathrm{CM}}$, where $D_{\mathrm{MLT}}$ is the diffusion coefficient for convective element mixing and $D_{\mathrm{CM}}$ that for composition mixing. Herwig (2001) finds that the rapid evolution of V4334 Sgr is accounted for by $f \sim 10-100$, and that, for this value of $f$, the surface ${ }^{12} \mathrm{C} /{ }^{13} \mathrm{C}$ ratio for the $f=30$ VLTP model is $\sim 5$. This is clearly very much in line with the results presented here.

Pavlenko \& Geballe (2002) found that dust made a major contribution to the emission longward of $2 \mu \mathrm{m}$ in 1998 , and indeed was present as early as 1997 . The value of the parameter $\epsilon_{0}$ gives an indication of the level of the dust emission at $2.3 \mu \mathrm{m}$. From Table 1 we see that $\epsilon_{0} \simeq 2 \pm 0.2$, indicating that the dust flux at $2.3 \mu \mathrm{m}$ is $\simeq 9.4 \times 10^{-12} \mathrm{~W} \mathrm{~m}^{-2} \mu \mathrm{m}^{-1}$. This is much higher than in later spectra, when the star had become completely obscured and the dust emission dominated the overall spectrum (Tyne et al. 2002). At later times ( 21999$)$ the dust envelope expanded and cooled and the peak of its spectrum shifted to longer wavelengths.

\section{Conclusion}

We have carried out a spectral synthesis to determine the ${ }^{12} \mathrm{C} /{ }^{13} \mathrm{C}$ ratio in $\mathrm{V} 4334 \mathrm{Sgr}$. Given a range of $\mathrm{C}$ abundance, we determine that ${ }^{12} \mathrm{C} /{ }^{13} \mathrm{C} \simeq 4 \pm 1$, consistent with the VLTP interpretation of $\mathrm{V} 4334 \mathrm{Sgr}$. The relative dust contribution at $2.3 \mu \mathrm{m}$ is $\epsilon_{0} \simeq 2 \pm 0.2$. Our value of the ${ }^{12} \mathrm{C} /{ }^{13} \mathrm{C}$ ratio is quite well constrained, and will doubtless be useful in fine-tuning the VLTP scenario.

Acknowledgements. The UK Infrared Telescope is operated by the Joint Astronomy Centre on behalf of Particle Physics and Astronomy Research Council (PPARC). T.R.G. is supported by the Gemini Observatory, which is operated by the Association of Universities for Research in Astronomy, Inc., on behalf of the international Gemini 

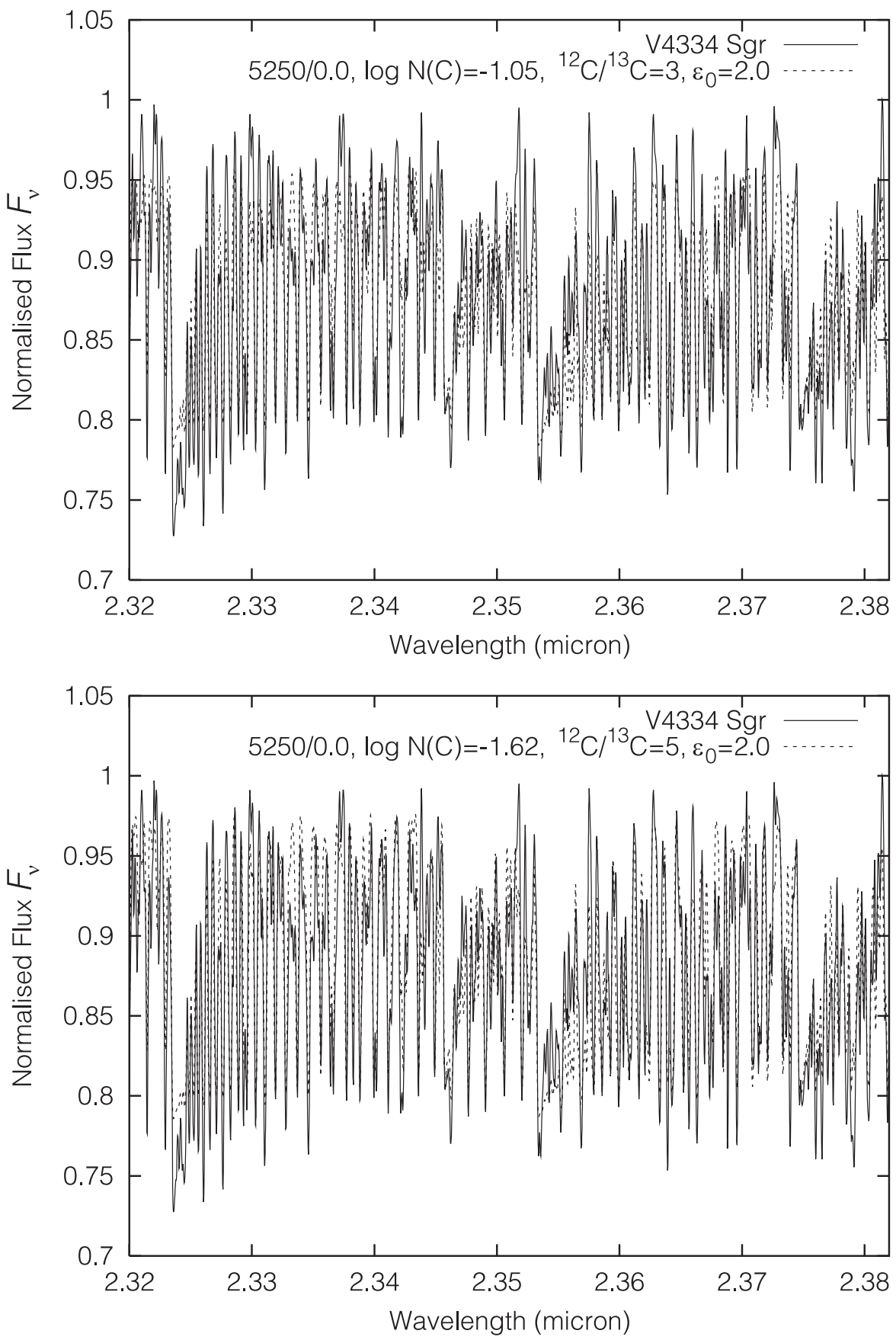

Fig. 3. a) Best fit to 1998 July spectrum found by the minimisation procedure outlined in the text, for $\log N($ C) $=-1.05$. b) As a), but for $\log N(\mathrm{C})=-1.62$.
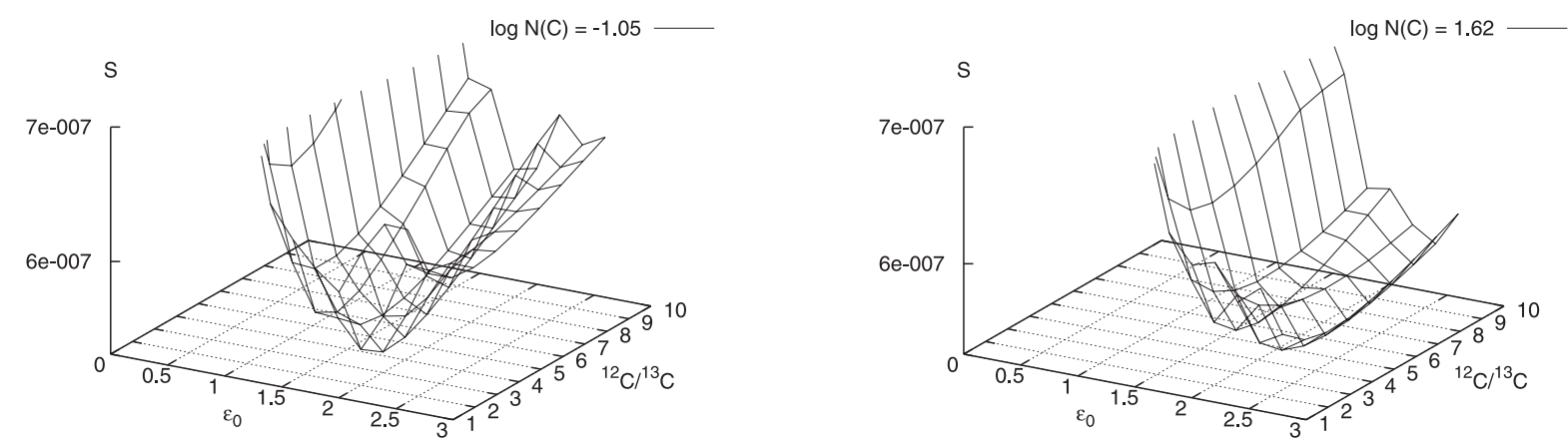

Fig. 4. a) Dependence of the fit parameter $S$ on ${ }^{12} \mathrm{C} /{ }^{13} \mathrm{C}$ and $\epsilon_{0}$ for a grid of the best fits found by minimisation procedure for $\log N(\mathrm{C})=-1.05$. b) As a), but for $\log N(\mathrm{C})=-1.62$. 
partnership of Argentina, Australia, Brazil, Canada, Chile, UK and USA. This work was partially supported by a PPARC visitors grants from PPARC and the Royal Society. Y.P.'s studies are partially supported by a Small Research Grant from American Astronomical Society. This research has made use of the SIMBAD database, operated at CDS, Strasbourg, France.

\section{References}

Asplund, M., Lambert, D. L., Kipper, T., Pollacco, D., \& Shetrone, M. D. 1999, A\&A, 343, 507

Dürbeck, H. W., \& Benetti, S. 1996, ApJ, 307, L111

Goorvitch, D. 1994, ApJS, 95, 535

Herwig, F. 2001, ApJ, 554, L71

Jones, H. R. A., Pavlenko, Ya., Viti, S., \& Tennyson, J. 2002, MNRAS, 330,675

Kerber, F., Blommaert, J. A. D. L., Groenewegen, M. A. T., et al. 1999, A\&A, 350, 27

Kipper, T. 1999, Baltic Astron., 8, 483

Kupka, F., Piskunov, N., Ryabchikova, T. A., Stempels, H. C., \& Weiss, W. W. 1999, A\&AS, 138, 119
Kurucz, R. L. 1993, CD ROM 9, 18, Harvard-Smisthonian Observatory

Lawlor, T. M., \& MacDonald, J. 2003, ApJ, 583, 913

Mountain, C. M., Robertson, D. J., Lee, T. J., \& Wade, R. 1990, SPIE, 1235,25

Pavlenko, Y. V. 1997, Astron. Rep., 41, 537

Pavlenko, Ya. V. 2002, Ap\&SS, 279, 91

Pavlenko, Ya. V., \& Dürbeck, H. W. 2001, A\&A, 367, 993

Pavlenko, Ya. V., \& Geballe, T. R. 2002, A\&A, 390, 621

Pavlenko, Ya. V., \& Jones, H. R. A. 2002, A\&A, 396, 967

Pavlenko, Ya. V., \& Zhukovska, N. S. 2003, Kinematika i Fizika Nebesnykh Tel., 19, 28

Pavlenko, Ya. V., Jones, H. R. A., \& Longmore, A. J. 2003b, MNRAS, 345,311

Seaton, M. J., Zeippen, C. J., Tully, J. A., et al. 1992, Rev. Mex. Astron. Astrophys., 23, 107

Tyne, V. H., Eyres, S. P. S., Geballe, T. R., et al. 2000, MNRAS, 315, 595

Tyne, V. H., Evans A., Geballe, T. R., et al. 2002, MNRAS, 334, 875

Unsöld, A. 1955, Physik der Sternatmospheren, 2nd ed. (Berlin: Springer) 